
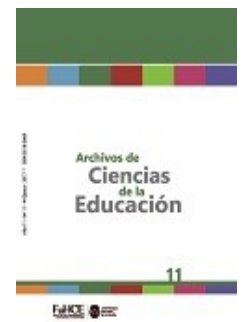

Archivos de Ciencias de la Educación, Vol 11, n 11, 2017, e020. ISSN 2346-8866

Universidad Nacional de La Plata.

Facultad de Humanidades y Ciencias de la Educación.

Departamento de Ciencias de la Educación.

\section{Un modo de enseñar Didáctica en la formación de profesores}

\author{
A way of teaching Didactics in teacher training
}

\section{Victoria Baraldi}

Universidad Nacional Litoral, Argentina | vbaraldi@gmail.com

\section{PALABRAS CLAVE}

Didáctica

Enseñanza

Formación docente

Curriculum

\section{KEYWORDS}

Didactis

Teaching

Teacher'sformation

Curriculum

\section{RESUMEN}

El presente trabajo describe un modo de enseñar Didáctica en el marco de la formación de profesores universitarios. Para ello se mencionan los planes de estudios en los que se incluye la cátedra Didáctica General, los principales conceptos y principios que dan sentido al programa y los ejes con los que se trabaja; al mismo tiempo, se comparte un conjunto de movimientos, preguntas e inquietudes que su puesta en marcha genera en los estudiantes. Sobre la base de lo expuesto, se fundamenta que el corpus que se selecciona para ser enseñado es producto de un trabajo articulado entre enseñanza, investigación y extensión, a través de cuyas prácticas fue posible plantear preguntas, trabajar con otros, aprender de los errores y los aciertos. Esto ocurre en el marco de un proceso abierto y colaborativo de un equipo de cátedra de una universidad pública argentina, en este caso, la Universidad Nacional del Litoral.

\section{ABSTRACT}

This work describes a way to teach Didactics within the framework of the university teacher's formation. For that, a curriculum is mentioned in which "General Didactics" is included, the main concepts and principles that give shape to the program, the core ideas to work with, and at the same time a combination of movements is shared, questions and worries generated among students through its launching. On the basis of what is set out, the chosen corpus to be taught is a consequence of an articulated work of teaching, research and extension, and through which its practices it was possible to make questions, work with others, and learn from the right and wrongs. This takes place within an open and collaborative process of a public university team from Argentina, in this particular case, The National University from the Litoral (Universidad Nacional del Litoral in Spanish). 


\section{¿Por qué este texto?}

En las últimas décadas el campo de la Didáctica ha sido ámbito de debates, indagaciones, mutaciones y controversias. Luego de la última dictadura militar, las perspectivas que se centraban sólo en cuestiones técnicas fueron criticadas y revisadas, y adoptaron mayor presencia aquellas otras que enfatizaron la dimensión política de la enseñanza e hicieron profundos análisis acerca de lo que se definía para ser enseñado. De la mano de autores norteamericanos como Henry Giroux y Michael Apple se volvieron a leer pedagogos críticos de nuestra región y se recuperaron textos, como fue el caso de Paulo Freire y de pedagogos argentinos que habían sido prohibidos. Las perspectivas coexisten en versiones aggiornadas, a pesar de sus disidencias, no se puede afirmar que una perspectiva haya sustituido a la otra.

Los debates incluyeron la discusión en torno al estatuto científico de la Didáctica, que fuera cuestionado desde una visión positivista. Los argumentos en su defensa, basados en las especificidades de las ciencias sociales, no tardaron en aparecer (Camilloni, 1994). Otros asuntos también fueron motivo de debate como los vínculos y desvínculos entre concepciones generales y específicas de la Didáctica; los modos en que históricamente se construyó conocimiento (Díaz Barriga, 2000); la necesidad de una pluralidad metodológica para la investigación, que ya había sido señalada por Klafki (1986) y reforzada por otros pedagogos; las relaciones entre teorías y prácticas (Basabe, 2007) y el uso crítico de la teoría (Zemelman, 2006).

A su vez la Didáctica, ciencia de encrucijada como dijera Camilloni (1996), siempre se nutrió de otras disciplinas y en el siglo XX entabló relaciones con otros campos de conocimiento, que primero emergieron realizando un desplazamiento -como sucedió con el campo del curriculum y el de la tecnología educativa-, pero que luego quedaron incorporados y/o fuertemente relacionados con el campo de la Didáctica.

Por otra parte, distintos programas de investigación se desarrollaron a partir de detectar la imposibilidad en algunos estudiantes para hacer un uso activo del conocimiento. Uno de los más importantes fue el de enseñanza parala comprensión del Proyecto Cero generado en Estados Unidos (Stone Wiske, 1999) con fuerte resonancia en Argentina. Desde otras referencias teóricas, una línea de indagación estudia la enseñanza con relación a saberes socialmente productivos (Puiggrós y Gagliano, 2004) y aprendizajes socialmente significativos (Orozco,2009).

Es así que en el campo de la Didáctica hay acuerdos parciales y muchas discusiones, y algunos temas tienen programas de investigación más desarrollados y otros más incipientes. Del significativo acervo bibliográfico hemos hecho referencia sólo a un pequeño grupo de autores y de textos pues ante la propuesta de escribir un texto sobre Didáctica, en vez de desarrollar algunos de estos temas en debate decidí compartir un modo de enseñar Didáctica y dialogar así en torno a las prácticas de la enseñanza de esta disciplina. La opción realizada exige objetivar decisiones y recorridos, los que se encuentran implícita o explícitamente enunciados al describir un escenario particular. Éste es el propósito del texto, compartir una forma de enseñar Didáctica en el marco de un proceso de formación docente en una universidad pública de nuestro país.

Los principios de situacionalidad y singularidad echan por tierra que esta descripción se convierta en modelo o en estéril intento de réplica. Es solo una ocasión para dialogar. Para que este diálogo posterior sea posible, el texto se organiza de la siguiente manera: primero se mencionan 
componentes de los planes de estudio en los que se desarrolla la cátedra de Didáctica General, luego se analizan supuestos y principios pedagógicos que la sustentan y los ejes que organizan el programa, para finalizar se describe el modo de trabajo que posibilitó la definición y continuidad de la propuesta pedagógica.

\section{5 semanas...}

Casi cuatro meses es el tiempo cronológico asignado a Didáctica General, asignatura del eje de la Formación Pedagógica en los profesorados con sede en la Facultad de Humanidades y Ciencias de la Universidad Nacional del Litoral. De acuerdo a los planes de estudio vigentes, los estudiantes inician el cursado de la misma cuando ya regularizaron Sociología de la Educación y Psicología de la Educación; pudiendo hacerlo de modo simultáneo con Política Educativa. Este trayecto de formación pedagógica se continúa con una Didáctica Específica y la Práctica Docente correspondiente al profesorado que eligieron: Historia, Geografía, Matemática, Letras, Biología, Filosofía o Química. La duración de estas carreras (según los planes de estudio) es de cinco años y cursan, simultáneamente a la formación pedagógica, las asignaturas de las disciplinas específicas que reúnen la mayor carga horaria. También tienen asignadas dos materias de formación general que eligen entre Filosofía, Psicología y Sociología. Al egresar quedan habilitados para ejercer la docencia en los niveles secundario y superior. Una de las riquezas que hace al funcionamiento de la asignatura Didáctica General, es que estudiantes provenientes de todos los profesorados mencionados asisten a ese mismo espacio de formación.

El carácter cuatrimestral de la materia -que devino de un proceso de revisión de los planes de estudio basado en la equívoca ilusión de que esto acortaría el tiempo de duración de las carrerasrequiere de una clara definición de los contenidos y las actividades para que representen procesos significativos para los estudiantes y sean viables en su implementación.

Somos conscientes del carácter ficcional de una asignación temporal establecida en semanas y horas de cursado, ya que esto es un parámetro externo al proceso de enseñanza. Por el contrario, reconocemos la complejidad del tiempo didáctico ${ }^{1}$ que se dirime sobre la base del registro de múltiples temporalidades que se conjugan -o colisionan- en la práctica de la enseñanza. El proceso reconstructivo del saber del docente a partir del cual anticipa y proyecta una propuesta de enseñanza, luego se entrecruza con urgencias, sorpresas, recurrencias de otros tiempos subjetivos, institucionales y sociales al momento de su implementación. Esto requiere del docente revisiones y definiciones acordes a cada situación.

\section{Antes del viaje...otros viajes}

En varias oportunidades hemos recurrido a la metáfora del viaje para representar el trayecto de formación que implica cursar Didáctica General. A dicha metáfora la hemos utilizado con propósitos evaluativos al finalizar el cuatrimestre, en donde solicitamos a los estudiantes que reconstruyan -también metafóricamente- este trayecto dando cuenta de las principales estaciones, los imprevistos, las bifurcaciones, las compañías, el equipaje y los recuerdos.

La definición de nuestra propuesta de enseñanza también implicó el pasaje por varias estaciones. En 
parte, tuvimos que olvidar lo aprendido - ¿y si aprender fuera olvidar?, nos invitó a pensar Ospina (2010)-, formular y sostener preguntas para desarrollar proyectos de investigación, dialogar con otros docentes, releer los clásicos -así como también conocer las nuevas investigaciones- y adentrarnos en otros campos de conocimiento para luego volver a la enseñanza. Como nos lo recordara Camilloni, formamos parte de una disciplina que "está y estará siempre en camino de construirse y reconstruirse en razón de la naturaleza, en algunos aspectos inevitablemente incierta, de la enseñanza, su objeto de estudio” (2007, p.15); un objeto que está atravesado por dimensiones teóricas, históricas y políticas.

\section{Definiciones pedagógicas}

Cuando años atrás nos preguntábamos cuáles serían los conceptos, las formas de razonamiento y las prácticas que potenciarían a los futuros docentes hacia una concientización creciente sobre la complejidad del presente, volvimos a leer pedagogos de la talla de Simón Rodríguez y Paulo Freire; y analizamos un conjunto de experiencias educativas alternativas para poder responder al interrogante planteado. En esta búsqueda y sobre la base del trabajo en las aulas, formulamos principios que orientaron y orientan nuestra perspectiva pedagógica: “vincular los procesos de enseñanza con procesos sociales contemporáneos; generar reflexividad en los procesos de formación; trabajar entre instituciones y entre disciplinas; recuperar la imaginación en los espacios de formación” (Baraldi, Bernik y Díaz, 2012, p.129).

Siempre nos preocupó que el cursado de nuestra asignatura no sea parte de una carrera de obstáculos para recibirse, sino una experiencia de formación. Es decir, un trabajo en torno a sí mismo, lo cual en este caso implica revisar y cuestionar el propio vínculo con el conocimiento, las concepciones heredadas, las prácticas de estudio y los modos en que nos posicionamos en los procesos de enseñar y de aprender.

Para que esto sea posible, además de los principios mencionados, otras dos cuestiones han surcado nuestro programa: la atención y revisión de las prácticas de lectura y de escritura, y la inmersión en situaciones educativas que requieren de los estudiantes la toma de decisiones sobre la enseñanza. Con respecto a la primera cuestión, y a raíz de un trabajo de adscripción en docencia (Monserrat, 2009) nos hicimos eco de los trabajos en torno a la alfabetización académica. $\stackrel{2}{\text { Allí nos }}$ interrogamos sobre las prácticas de lectura y de escritura en los procesos de formación y su incidencia en la conformación de la subjetividad de quien se está formando como docente. Estos interrogantes derivaron no sólo en la revisión de los criterios para la selección del corpusbibliográfico del programa sino también en la elaboración de estrategias de acompañamiento que implicaran otros modos de leer.

También nos preguntamos sobre las prácticas de escritura que hacen al trabajo docente, cuáles son las más frecuentes y cuáles otras serían necesarias para fortalecer un trabajo intelectual. No se trata sólo de saber qué dice un autor, sino a quién, dónde y por qué dice lo que dice, y qué puedo pensar -y escribir- sobre eso que leí. La secuencia leer-pensar-escribir permite correrse de la visión de la escritura como acción de reproducción simplificada de lo leído -acción muy frecuente en los ámbitos académicos- y habilitar otras posibilidades que permitan expresar y comunicar los propios pensamientos. Esas definiciones implicaron desde entonces, la asignación de tiempos específicos 
para acompañar nuevos procesos en aras de fortalecer, desde ese ángulo, el trabajo intelectual del docente, cometido central de nuestra propuesta de enseñanza.

La segunda cuestión apunta al trabajo deliberativo, indispensable al formar en una disciplina con un fuerte componente de orden práctico como es la Didáctica. En distintos ensayos e investigaciones se ha podido fundamentar que la mejora de la práctica docente es posible si hay modificaciones en los razonamientos prácticos de los profesores. El movimiento inglés iniciado a fines de la década del '60 que definía a los profesores como investigadores se apoya en este principio. La implementación de cada uno de los proyectos en el marco de esa perspectiva de trabajo, parte del supuesto de que la mejora de la práctica se sustenta en el cambio de concepciones en los educadores. $\underline{3}$ Esto nos remite a lo dicho por Dewey cuando afirmaba que "la realidad final de la ciencia de la educación no se encuentra en los libros, ni en los laboratorios experimentales, ni en las salas de clase donde se enseña, sino en las mentes de los que dirigen las actividades educativas” (1960, p.35). Más cercano en el tiempo, y en el marco de una discusión acerca de las metodologías utilizadas para la investigación de la enseñanza, Fenstermacher afirma que "el beneficio de la investigación sobre educación para la práctica educativa se realiza en el perfeccionamiento de los razonamientos prácticos, no en programas de actividades deducidos de los resultados de la investigación” (1989, p.165).Y más recientemente nos lo recuerda Basabe en las siguientes afirmaciones: “(...) la didáctica es una teoría comprometida de manera directa con la mejora de las prácticas de enseñanza, pero su intervención sobre ellas siempre es mediada a través del juicio práctico de los practicantes”(2007, p.227) y “si el horizonte normativo se termina de delinear a partir del juicio práctico de los practicantes, la didáctica debe orientarse a ofrecer una plataforma productiva para la deliberación y la toma de decisiones” (2007, p.221).

Éstas son las razones por las cuales nuestra propuesta de enseñanza no consiste sólo en el conocimiento de categorías propias del campo disciplinar, absolutamente necesarias para comprender este fenómeno complejo, ni en el reconocimiento de juicios normativos que puedan orientar una práctica. Con el convencimiento de que ambas cuestiones son importantes, pensamos que también se requiere la inmersión en situaciones prácticas, ya que en ellas tienen lugar las decisiones que ponen de manifiesto nuestras comprensiones.

Esta apuesta requirió de un trabajo en sintonía con escuelas secundarias de la ciudad de Santa Fe. En algunos casos, enmarcados en proyectos de extensión, y más recientemente, en un nuevo dispositivo de la UNL denominado Prácticas de Extensión de educación experiencial tendiente a la incorporación de la extensión en la formación de grado. Este proyecto se inició hace una década y ha sido acompañado por distintas instancias institucionales, tales como cursos, jornadas de divulgación, para concretar su implementación. $\underline{4}$

En el dispositivo arriba mencionado y adaptado a nuestra asignatura, los estudiantes participan en situaciones de enseñanza disciplinar o en proyectos institucionales que implican la confluencia de distintas asignaturas. Allí colaboran en la selección de materiales educativos y en la implementación de actividades, algunas de ellas de carácter lúdico. También ayudan a estudiantes que manifiestan dificultades y, especialmente, participan en la elaboración de propuestas -muchas de ellas innovadoras- para la implementación de proyectos institucionales.

Este trabajo realizado con las escuelas secundarias, nos permitió construir un vínculo diferente al 
que desde el nivel superior se establecía con los otros niveles del sistema educativo centrado en asistir para observar, clasificar y juzgar lo observado. Por el contrario, la presencia en las escuelas apuntó a conocer para comprender y colaborar. A su vez, las planificaciones extremadamente analíticas y codificadas que hacían los estudiantes en otras épocas, son sustituidas por guiones conjeturales (Bombini, 2001) que se constituyen en oportunidades de revisión de sus comprensiones respecto de la práctica de enseñanza.

\section{Ejes que organizan el programa}

Planteamos la idea de ejes, en este caso tres, en tanto ángulos de observación. Si bien todo análisis implica una separación, aquí se realiza con el propósito de distinguir para conjugar (Morin, 1998). Cada eje reconstruye e integra los previos y ello se evidencia en el modo de presentarlos, en la definición de actividades y en la evaluación. En estos ejes analizamos: la Didáctica y su objeto de conocimiento, la cuestión curricular -para que se pueda visualizar la enseñanza de la disciplina en el marco de un proyecto educativo-, y el proceso de construcción metodológica entendido como síntesis de opciones. En la última versión del programa los denominamos así "I. Los desafíos de la enseñanza como práctica social; II. La construcción del objeto de enseñanza; III. La elaboración de propuestas de enseñanza” (Baraldi, Bernik, Díaz, Grinóvero, 2016). En los próximos tres subtítulos daremos cuenta de aspectos centrales abordados en cada uno de ellos. $\underline{5}$

\section{Primera estación}

Una de las particularidades que tienen las ciencias sociales, es que sus términos forman parte del lenguaje cotidiano. Esto para algunos es una “maldición” y para otros se trata de la existencia de más conceptos que términos. Lo cierto es que se torna ineludible darles a las palabras el peso específico que tienen en cada campo disciplinar, despojarlas de su sentido común, advertir la carga ideológica con las que pasan a formar parte de nuestras representaciones y también reconocer el carácter polisémico que los términos tienen en ciencias sociales.

Enseñanza, Didáctica, formación, son las primeras categorías que ponemos en cuestión. Trabajamos en torno al concepto genérico de enseñanza (Fenstermacher, 1989) y sobre la diferencia entre los procesos de enseñanza y de aprendizaje -los cuales se relacionan desde un punto de vista ontológico y no causal-. También analizamos el concepto de buena enseñanza,por la relevancia dada a las dimensiones epistemológicas y morales, e identificamos las particularidades de la enseñanza en instituciones educativas (Basabe y Cols, 2007). Reconocer la especificidad y complejidad de la enseñanza permite comprender a su vez la particularidad de la Didáctica que construye teorías sobre esta práctica.

En cuanto a los debates epistemológicos, por el tiempo del cursado, sólo realizamos una suerte de “cartografía” de algunas perspectivas, referenciando un conjunto de autores clásicos que dieron respuestas a los problemas y encrucijadas de su tiempo y con vigencia en la actualidad. Al analizar sus perspectivas es posible reconocer el carácter teórico, histórico y político de la Didáctica.

Muchas veces nos hemos preguntado si el corpus bibliográfico de la asignatura no tendría que estar conformado solo o principalmente por textos clásicos a partir de los cuales realizar una lectura 
culturalmente situada (Casalla, 1977). $\underline{6}$ No obstante, consideramos aceptable realizar breves exposiciones, analizar el paratexto de libros "emblemáticos", compartir videos, leer y comentar párrafos seleccionados. No pretendemos que los estudiantes sean especialistas en estos autores; ${ }^{7}$ - sino que conozcan de su existencia -porque es el único espacio de la carrera en donde se los nombra- y que adviertan que, al enseñar su disciplina, también tendrán que hacer -al igual que ellos y nosotras- opciones teóricas.

Para qué enseñar la disciplina (historia, geografía, letras, matemática, química, biología, filosofía) es la pregunta que pretendemos instaurar en este primer eje. Consideramos que, para dar respuesta a este interrogante, no basta con conocer la disciplina, sino que para enseñar cuestiones en torno a ese campo de estudio en la escuela es necesario preguntarse por el sentido social de la profesión y por el tipo de sujeto que se pretende formar. Sobre este punto escuchamos las preocupaciones de los estudiantes y también compartimos las nuestras. Más allá de ciertas especificidades de los contextos en los que tenga lugar la enseñanza, expresamos nuestra preocupación sobre rasgos de esta época. Rasgos que nos atraviesan, nos interpelan y nos exigen posicionarnos en torno al sentido de la educación, tales como: los procesos de concentración de la riqueza y la expansión de la pobreza, la transnacionalización de la economía y de los medios de comunicación, la creciente celeridad de los procesos, los cambios en los modos en que circula la información y el conocimiento, el deterioro ambiental, la invisibilidad de algunos sectores sociales, la cuestión de la memoria, la paz y la justicia.

Luego de las primeras semanas, los estudiantes revisan sus expectativas iniciales sobre esta asignatura a la cual asistieron con la idea de que "les iban a decir cómo hay que enseñar". Distinguen con mayor claridad los procesos de enseñar y de aprender y se cuestionan acerca del sentido de la enseñanza. Algunos advierten un vacío de reflexiones epistemológicas sobre el campo de estudio disciplinar del profesorado que cada uno cursa y sobre cuestiones sociales actuales. También expresan -a veces al final del cuatrimestre- que es en Didáctica General donde comienzan a "pensarse como docentes".

\section{No estamos solos}

En el segundo eje enfatizamos sobre la cuestión curricular. Pretendemos que visualicen la enseñanza de su disciplina en el marco de un proyecto educativo. Tomamos como principal referencia a de Alba (1991) quien enfatiza las dimensiones histórico-políticas y ofrece instrumentos que consideramos apropiados para analizar un objeto tan complejo como es el curriculum. También aludimos a otros aportes como el curriculum como cerco cognitivo (Frigerio, 1992), los ámbitos de referencia (Terigi, 2004) y la transposición didáctica (Chevallard, 1991). Las nociones de procesos de construcción curricular y saberes del docente (Baraldi y Morzán, 2013) son planteados como conceptos para reconocer el carácter dinámico y participativo que, en algunos casos, pueden tener los procesos curriculares. También se analizan desde distintos autores las categorías de curriculum oculto y curriculum nulo en tanto permiten develar prácticas de conocimiento no suficientemente visualizadas o ausentes.

Para la comprensión de la cuestión curricular, además de la lectura de bibliografía específica, los estudiantes analizan documentos curriculares cuyo corpus varía de acuerdo a las políticas 
educativas nacionales y jurisdiccionales vigentes. También conocen proyectos educativos institucionales de escuelas secundarias de la ciudad de Santa Fe y realizan entrevistas a sujetos de desarrollo curricular: directivos, docentes y estudiantes.

Para muchos de ellos, es la primera vez que escuchan la noción de curriculum y reconocen su importancia, en tanto partían de una idea -bastante instalada, por cierto- de considerar al contenido como un legado dado por el docente a quien van a reemplazar. Esto nos recuerda lo señalado por Apple (1986) cuando además de hacer evidente las características de transmisión de la ciencia en los ámbitos escolares, señala la tendencia a que los hombres y mujeres se vean más como receptores de las normas y no como constructores de las mismas. En este sentido, la noción de sujetos de determinación y estructuración curricular permite revisar este imaginario, y la categoría saberes del docente (Baraldi y Monserrat, 2014) es analizada como condición de posibilidad de procesos dinámicos y participativos.

\section{Con las manos en la masa}

A la hora de tratar el proceso de construcción metodológica (Edelstein, 1996) ya hemos “despejado" -al menos así lo creemos- la idea de que la enseñanza se "resuelve” con la incorporación de técnicas y recursos novedosos para entusiasmar a los alumnos. Desde el concepto de buena enseñanza ya se ha analizado que toda propuesta se constituye sobre la base de decisiones éticas y epistemológicas definidas por el tipo de sujeto que se quiere formar. En esta instancia los estudiantes concurren semanalmente a escuelas de la ciudad de Santa Fe y su inserción tiene el propósito de colaborar en la implementación de un proyecto educativo, ya sea en el marco de una asignatura o en un proyecto institucional que requiere la presencia de distintos docentes.

En este eje volvemos a analizar la cuestión del contenido (fuentes, criterios de selección y organización), de los materiales educativos y la evaluación. Más allá de la profundidad y riqueza del corpus bibliográfico ofrecido para abordar estos tópicos, hay dos categorías muy potentes para el desarrollo de este eje y para las prácticas de extensión. Una es la noción de saberes socialmente productivos (Puiggrós y Gagliano, 2004) en tanto saberes que modifican a los sujetos enseñándoles a transformar la naturaleza y la cultura; y la otra -que sintoniza en sus bases epistemológicas y políticas-es la de aprendizajes socialmente significativos(Orozco, 2009) que enfatiza la dimensión política-cultural de los aprendizajes, incorpora los saberes producidos a través de la experiencia y articula prácticas escolares con prácticas comunitarias, sociales, culturales y productivas.

También las nociones de transposición didáctica y vigilancia epistemológica (Chevallard, 1991), el guión conjetural (Bombini,2001), la reflexión en torno al uso de las tecnologías (Litwin,2005) y las funciones e instrumentos de evaluación (Camilloni, 2015), configuran marcos de referencia importantes para involucrarse en esta breve pero significativa experiencia de enseñanza en escuelas secundarias de la ciudad de Santa Fe.

Como se puede observar, este tercer eje del programa tiene dos referencias empíricas centrales: lo que acontece en las aulas de escuelas secundarias a las que asisten en el marco de las prácticas de educación experiencial y la propia cátedra de Didáctica General donde se realiza un meta-análisis de las clases y se evalúa la práctica de enseñanza de acuerdo a criterios explicitados en nuestro programa y comunicado el primer día de clase. 
Los procesos de participación de los estudiantes en la escuela secundaria son disímiles. Algunos lo transitan sin mayor implicación. Otros se involucran y dejan "huellas" muy valoradas por los docentes a cargo de los cursos. $\underline{8}$ No obstante, en todos los casos, implica una situación que los moviliza y permite comprender de otro modo la multidimensionalidad, simultaneidad e imprevisibilidad de la práctica docente (Jackson, 1992). A su vez, los diálogos y los vínculos que establecen con los jóvenes que asisten a las escuelas secundarias de la ciudad los hace revisar estereotipos y significaciones negativas con los que generalmente son representados los jóvenes en los medios de comunicación.

\section{La parte en el todo, un camino a seguir construyendo}

Entendemos a la docencia universitaria en sintonía con las funciones sustantivas de este nivel educativo: la enseñanza, la investigación y la extensión. Esto en palabras de Risiere Frondizi recordadas por Saleme (1997, p.145) al despedir a un grupo de graduados- puede entenderse como “investigación científica, sensibilidad social, preservación del patrimonio cultural y formación de profesionales”. Sin esta amplitud, la enseñanza en la universidad puede quedar en trivialidades y en la distribución de "retazos de cultura". Por el contrario, el fecundo proceso de retroalimentación de estas funciones y la apuesta a la conformación de un equipo de cátedra 9 han sido los motores que permitieron y permiten la mejora y consolidación de nuestra propuesta pedagógica. En algunos casos, los problemas de enseñanza dieron lugar a la generación de proyectos de investigación, en otros, a proyectos de extensión, pero siempre ambos tipos de emprendimientos nutrieron los procesos de enseñanza (Baraldi, 2016b).

Todo lo que hemos enunciado hasta aquí forma parte de una perspectiva. Nuestro anhelo ha sido y es el formar un docente intelectual, que cuestione sus modos de comprender y de hacer, que desnaturalice las prácticas, que reaccione cuando el poder anula y minimiza. Que no pierda el rumbo de acuerdo al sentido que él le dio a su profesión. Que alimente su pasión, que sueñe, se interrogue y contagie entusiasmo. Que sepa que este mundo es una construcción y que otros mundos son posibles. Que la educación no lo puede todo, pero algo sí puede la educación. Que no pierda de vista quién es...y que sepa que no está solo.

Al cerrar estas páginas no puedo dejar de recordar algo afirmado al inicio: la enseñanza no causa el aprendizaje, lo busca, lo pretende. En tal sentido, quizá lo expresado hasta aquí sean anhelos e ilusiones que se visten de Didáctica para entrar en las aulas, y quizá este texto se construye de palabras que sólo ordenaron huellas y memorias para poder conversar sobre la enseñanza.

\section{Notas}

1 Sobre los modos en que se ha concebido el tiempo en distintos campos de conocimiento, y en particular en la Didáctica, he realizado la tesis doctoral: “Tiempo y didáctica. Itinerarios para pensar la enseñanza”, Facultad de Humanidades y Ciencias, Universidad Nacional del Litoral, Santa Fe, Argentina (Baraldi, 2016a). 
$\underline{2}$ Por aquel entonces Carlino (2005) ya nos había recordado a los docentes que también era nuestra tarea enseñar a leer y a escribir de acuerdo a los estándares académicos de la universidad.

$\underline{3}$ No olvidemos que el lema de Stenhouse, pedagogo que dirigió el Humanities Curriculum Proyect, era: “No es posible un desarrollo del currículum sin el desarrollo del profesor” (1987: 103).

4 Resolución UNL “C.S.” Nro. 274/07.

$\underline{5}$ No se realiza un análisis detallado de cada uno de ellos ni de su bibliografía en tanto el programa está disponible en la Facultad de Humanidades y Ciencias de la Universidad Nacional del Litoral en http://www.fhuc.unl.edu.ar/programas/programas imprimir.php?

nId Programa Materia=1013\&nId Lectivo=16

6Casalla plantea la idea de lectura culturalmente situada en tanto permite entrever la situación como "el horizonte de sentido contra y a partir del cual opera un determinado pensamiento o actividad” (1977:105).

$\underline{7}$ En general trabajamos con Comenio, Dewey, Tyler, Taba y Freire porque representan perspectivas centrales sobre la enseñanza y además porque en ellos se puede visualizar la función dada a la escuela y las concepciones de conocimiento, de sujeto y de cultura.

$\underline{8}$ Una síntesis de uno de los proyectos de extensión puede consultarse en el video que realizamos: Enseñar contenidos curriculares comunes. Un desafío para la universidad y la educación secundaria. Disponible en: http://www.fhuc.unl.edu.ar/pages/extension/programas-yproyectos/proyectos-de-extension/educacion.php

$\underline{9}$ Siempre hicimos alusión a la idea de que una cátedra es más que una asignatura. Didáctica General está integrada por Julia Bernik, Natalia Diaz, Nora Grinóvero y quien escribe. María Eugenia Stringhini y Mercedes Monserrat también formaron parte, pero luego optaron por el desempeño en otros ámbitos laborales.

\section{Bibliografía}

Apple, M. (1986). Ideología y curriculum. Madrid: Akal.

Baraldi, V. (2016a). Tiempo y didáctica: itinerarios para pensar la enseñanza. Paraná: Universidad Nacional de Entre Ríos.

Baraldi, V. (2016b). Enseñanza, Investigación y Extensión: Tres prácticas articuladas en la cátedra de Didáctica General de la UNL. E versión digital (6), 306-313

Baraldi, V., Bernik, J. y Díaz, N. (2012). Una didáctica para la formación docente. Dimensiones y principios para la enseñanza. Santa Fe: Ediciones UNL.

Baraldi, V. y Monserrat, M. (2014). La dimensión política de la enseñanza. Saberes del docente y procesos de construcción curricular. Novedades educativas (279), 84-88

Baraldi, V. y Morzán, A. (2013). Intersticios y saberes en los procesos de construcción curricular. 
Notas para pensar las prácticas docentes. Intersticios (4), 109-122

Baraldi, V.; Bernik, J.; Diaz, N. y Grinóvero, N. (2016). Programa de Cátedra de Didáctica General. Recuperado del sitio de la Facultad de Humanidades y Ciencias, Universidad Nacional del Litoral: $\quad$ http://www.fhuc.unl.edu.ar/programas/programas imprimir.php? nId Programa Materia=1013\&nId Lectivo=16

Basabe, L. (2007). Acerca de los usos de la teoría didáctica. En Camilloni, A. (Comp.) El saber didáctico (pp. 201-231). Buenos Aires: Paidós.

Basabe, L. y Cols, E. (2007). La enseñanza. En Camilloni, A.(Comp.) El saber didáctico (pp. 125161). Buenos Aires: Paidós.

Bombini, G. (2001). Prácticas docentes y escritura: hipótesis y experiencias en torno a una relación productiva. Ponencia presentada en el Congreso de Prácticas docentes. Facultad de Filosofía y Humanidades, Universidad Nacional de Córdoba. Córdoba, Argentina.

Camilloni, A. (1994). Epistemología de la didáctica de las ciencias sociales. En Aisenberg, B. y Alderoqui, S. (Comps.). Didáctica de las Ciencias Sociales. Aportes y reflexiones (pp. 25-41). Buenos Aires: Paidós.

Camilloni, A. (1996). De herencias, deudas y legados. En Camilloni, A. et al. Corrientes didácticas contemporáneas (pp. 17-39). Buenos Aires: Paidós.

Camilloni, A. (2015). La responsabilidad social y pedagógica de la evaluación de los aprendizajes. Itinerarios Educativos (8), 51-64.

Carlino, P. (2005). Escribir, leer y aprender en la universidad. Buenos Aires: Fondo de Cultura Económica.

Casalla, M. (1977). Crisis de Europa y reconstrucción del hombre. Buenos Aires: Castañeda.

Chevallard, Y. (1991). La transposición didáctica: del saber sabio al saber enseñado. Buenos Aires: Aique.

de Alba, A. (1991). Curriculum: crisis, mito y perspectiva. México: CESU-UNAM.

Dewey, J. (1960). La ciencia de la educación. Buenos Aires: Losada.

Díaz Barriga, Á. (2000). La investigación en el campo de la didáctica. Modelos históricos. En I Congreso Internacional de Educación "Educación, crisis y utopías". Facultad de Filosofía y Letras, Universidad Nacional de Buenos Aires, Argentina.

Edelstein, G. (1996). Un capítulo pendiente: el método en el debate didáctico contemporáneo. En: Camilloni, A. et al. Corrientes didácticas contemporáneas (pp.75-89). Buenos Aires: Paidós.

Fenstermacher, G. (1989). Tres aspectos de la filosofía de la investigación sobre laenseñanza. En Wittrock, M. La investigación de la enseñanza. Vol I. Enfoques, teorías y métodos (pp.149-179). Buenos Aires: Paidós.

Frigerio, G. (Comp.) (1992). Curriculum presente. Ciencia ausente: normas, teorías y críticas. Buenos Aires: Miño y Dávila. 
Jackson, P. (1992). La vida en las aulas. Madrid: Morata.

Klaffki, W. (1986). Los fundamentos de una didáctica crítico-constructiva. Revista de Educación (280), 37-79

Litwin, E. (2005). Tecnologías educativas en tiempos de internet. Buenos Aires: Amorrortu.

Monserrat, M. (2009). Informe de Adscripción en Docencia en Didáctica General. Facultad de Humanidades y Ciencias, Universidad Nacional del Litoral. Santa Fe, Argentina.

Morin, E. (1998). Introducción al pensamiento complejo. Barcelona: Gedisa.

Orozco, B. (2009). Saberes socialmente productivos y aprendizaje. Articulación didáctica pedagógica. En Gómez Sollano, M.(Coord.) Saberes socialmente productivos y educación. Contribuciones al debate (pp. 79-94). México: UNAM.

Ospina, W. (2010). Preguntas para una nueva educación. Conferencia inaugural en el Congreso Iberoamericano de Educación: Metas Educativas 2021. Buenos Aires, Argentina.

Puiggrós, A. y Gagliano, R. (2004). La fábrica del conocimiento. Los saberes socialmente productivos en América Latina. Rosario: Homo Sapiens.

Saleme, M. (1997). La Universidad. ¿Una manera de vivirla? En Decires. Córdoba: Narvaja.

Stenhouse, L. (1987). La investigación como base de la enseñanza. Madrid: Morata.

Stone Wiske, M. (Comp.) (1999). La enseñanza para la comprensión. Vinculación entre la investigación y la práctica. Buenos Aires: Paidós.

Terigi, F. (2004). Curriculum: Itinerarios para aprehender un territorio. Buenos Aires: Santillana.

Zemelman, H. (2006). El conocimiento como desafío posible. México: IPECAL. 